\title{
SPECTROSCOPY AND THERMOLUMINESCENCE $\mathrm{OF} \mathrm{LuAlO}_{3}$ :Ce
}

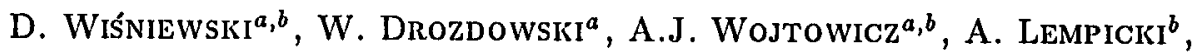 \\ P. Dorenbos ${ }^{c}$, J.T.M. DE HAAs ${ }^{c}$, C.W.E. van EIJK ${ }^{c}$ AND A.J.J. Bos ${ }^{d}$ \\ ${ }^{a}$ Inst. of Physics, N. Copernicus University, Grudziądzka 5, 87-100 Toruń, Poland \\ ${ }^{b}$ Dept. of Chemistry, Boston University \\ 590 Commonwealth Ave., Boston, MA 02215, USA \\ ${ }^{c}$ Delft Univ. of Tech., Faculty of Applied Physics, IRI \\ Mekelweg 15, 2629 JB Delft, The Netherlands \\ ${ }^{d}$ Delft Univ. of Tech., Interfaculty Reactor Inst., c.o. IRI \\ Mekelweg 15, 2629 JB Delft, The Netherlands
}

The present status of the $\mathrm{LuAlO}_{3}:$ Ce scintillator is reviewed. Scintillation mechanism of this material is based on capture by $\mathrm{Ce}^{3+}$ of holes and then electrons from their respective bands. Results of spectroscopic and thermoluminescence experiments are presented to support this model.

PACS numbers: $78.90 .+\mathrm{t}, 78.55 .-\mathrm{m}, 61.80 . \mathrm{Ed}, 29.40 .-\mathrm{n}$

\section{Introduction}

Scintillation properties of $\mathrm{LuAlO}_{3}: \mathrm{Ce}(\mathrm{LuAP})$ crystals were first reported by Lempicki et al. [1]. The interest in this material is derived from the high light yield (above 20000 photons $/ \mathrm{MeV}$ ), high density $\left(8.34 \mathrm{~g} / \mathrm{cm}^{3}\right.$ ) and short decay time (about $18 \mathrm{~ns}$ ). These properties result in one of the highest figures of merit of any known scintillator and put LuAP in the forefront when high counting rates, fast timing, good stopping power, and energy resolution are of importance $[1,2]$. Taking into consideration that this material is not fully optimized yet, and there is evidence that its performance may be highly improved [3,4], it becomes exceptionally desirable to understand the physics which governs its behavior.

\section{Materials and experiments}

LuAP samples were cut from boules grown by Litton Airtron by the Czochralski method. Detailed description of the growth process was presented in [1]. Crystals were optically clear and single-phase. Ce doped specimens were colorless with Ce concentrations 0.035 and 0.11 mole\% (as measured by spark source mass spectrometry). Color of nominally undoped specimens was slightly orange with level of unintended Ce contamination below $0.4 \times 10^{-4}$ mole\%. 
Excitation spectra were acquired using synchrotron radiation at Brookhaven National Synchrotron Light Source (BNSLS). Emission spectra were taken under optical (at BNSLS), $\gamma$-ray (100 $\mu \mathrm{C} \mathrm{Ru-Rh} \mathrm{source,} 0.5-2.9 \mathrm{MeV}$ ), and X-ray ( $8 \mathrm{keV}$ ) excitation. X-ray excited luminescence spectra were acquired using "transmission" geometry; sample was excited from one side, and emission gathered from the other one. Decays were obtained under pulsed synchrotron light at BNSLS. Thermoluminescence measurements were performed at Delft University, using facilities described in detail in [5] and [6]. Various sources were used for irradiation: ${ }^{60} \mathrm{Co}$ $\gamma$-source $(1.2 \mathrm{kGy} / \mathrm{h})$, X-ray tube with $\mathrm{Cu}$ anode $(3.6 \mathrm{kGy} / \mathrm{h})$ and IIg standard lamp.

\section{Experimental results and discussion}

Room temperature emission spectra of undoped and Ce-doped specimens, taken under $\gamma$ and X-ray excitation, are presented in Fig. 1. Trace $a$ shows the

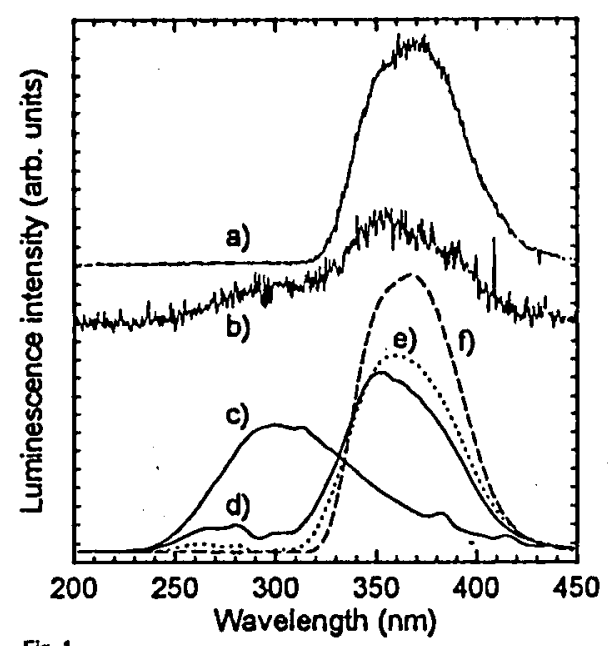

Fig. 1

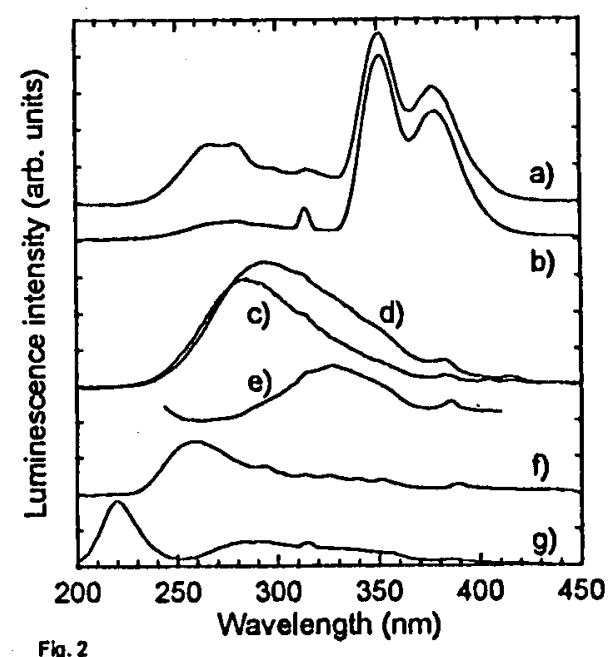

Fig. 2

Fig. 1. Room temperature emission spectra of LuAP under $\gamma(a, b)$ and X-ray $(c-f)$ excitation: $a-0.035$ mole\% Ce doped sample, $b$ and $c$ - undoped sample, $d-$ $0.035 \mathrm{~mole} \% \mathrm{Ce}$ doped sample, $0.5 \mathrm{~mm}$ thick, $e-0.035$ mole\% Ce doped sample, $1 \mathrm{~mm}$ thick, $f-0.11$ mole\% Ce doped sample, $1 \mathrm{~mm}$ thick.

Fig. 2. Luminescence spectra of 0.11 mole\% Ce doped $(a, b)$ and undoped $(f-g)$ LuAP samples under optical excitation: $a-161 \mathrm{~nm}, 13 \mathrm{~K}, b-153 \mathrm{~nm}, 13 \mathrm{~K}, c-161 \mathrm{~nm}$, $\mathrm{RT}, d-152 \mathrm{~nm}, \mathrm{RT} e-217 \mathrm{~nm}, 13 \mathrm{~K}, f-180 \mathrm{~nm}, 13 \mathrm{~K}$, and $g-148 \mathrm{~nm}, 13 \mathrm{~K}$.

$\gamma$-excited luminescence of Ce doped (0.035 mole\%) sample. This spectrum is dominated by a single band centered at about $365 \mathrm{~nm}$, which is a clear case of $\mathrm{Ce}^{3+}$ $d-f$ emission [1]. However, after more detailed examination, one can find an additional (about two orders of magnitude weaker) band at about $280 \mathrm{~nm}$. This band is somewhat stronger in case of nominally undoped sample $b$, but yet scintillation 
of this sample also consists mostly of $\mathrm{Ce}^{3+}$ emission. X-ray excited luminescence of the same sample $c$, on the contrary, does not demonstrate distinguishable cerium emission, showing instead an intense, wide, asymmetrical band peaking at about $300 \mathrm{~nm}$ (host emission), $\mathrm{Gd}^{3+}$ line at $314 \mathrm{~nm}$ and some $\mathrm{Tb}^{3+}$ lines. It is worth noting that $\mathrm{Gd}$ and $\mathrm{Tb}$ contaminations in this material are only 0.7 and 0.2 weight ppm, respectively, which suggest efficient energy transfer to these ions. X-ray excited luminescence spectrum of Ce-doped sample $d$ shows intense $\mathrm{Ce}^{3+}$ emission and some remains of $300 \mathrm{~nm}$ band. One can observe dips in host emission band at 275,292 and $310 \mathrm{~nm}$ which clearly correspond to bands in $\mathrm{Ce}^{3+}$ luminescence excitation spectrum (presented in Fig. 3). Ilost emission diminishes with increasing sample thickness (e) and Ce content; it vanishes almost completely for sample doped with 0.11 mole\% of $\mathrm{Ce}(f)$. These observations leave no doubt that cerium absorption is responsible for deformation of $300 \mathrm{~nm}$ band. Although it may indicate of nomradiative energy transfer from host emission to $\mathrm{Ce}^{3+}$ ions, we will show later that scintillation mechanism of LuAP:Ce is not based on this process.

Luminescence spectra under selective optical excitation are presented in Fig. 2. Traces $a$ and $b$ present low temperature spectra of 0.11 mole\% Ce doped sample excited by 160 and $153 \mathrm{~nm}$ light, respectively. Both spectra display host as well as $\mathrm{Ce}^{3+}$ emission, but their relative intensities depend on the excitation wavelength. Despite of surface excitation, an effect of deformation of host emission by cerium absorption is also evident, particularly in the first spectrum. Under $153 \mathrm{~nm}$ excitation a $\mathrm{Gd}^{3+}$ emission line at $314 \mathrm{~nm}$ is also observable. Room temperature (RT) spectra of the same sample will not be presented here since they do not reveal any additional features, except that splitting of $\mathrm{Ce}^{3+} \operatorname{doublet}\left({ }^{2} F_{5 / 2}\right.$ and ${ }^{2} F_{7 / 2}$ ) is no longer observed. Luminescence spectra of undoped sample let us see an undistorted shape of host emission, which in fact appears to be superposition of a few different ones overlapping each other. Excitation at around $160 \mathrm{~nm}$ reveals the strongest of these emissions peaking at about $280 \mathrm{~nm}(13 \mathrm{~K})$ and at $285 \mathrm{~nm}$ (RT) (c). Decreasing the excitation wavelength to $152 \mathrm{~nm}$ results in admixture of an additional emission on the long-wavelength side of $285 \mathrm{~nm}$ band $(d)$. At $13 \mathrm{~K}$, using $217 \mathrm{~nm}$ excitation light, it becomes possible to isolate this new emission as a band centered at about $330 \mathrm{~nm}(e)$. Furthermore, at low temperature one can obtain two extra emissions which are not visible otherwise. An excitation at $180 \mathrm{~nm}$ exposes an emission peaking at about $260 \mathrm{~nm}(f)$ and at $148 \mathrm{~nm}$ results in an emission band at $220 \mathrm{~nm}(g)$. Both emissions are about an order of magnitude weaker than $280 \mathrm{~nm}$ band, but while the $220 \mathrm{~nm}$ band is observable in undoped as well as in intentionally Ce doped samples, the $260 \mathrm{~nm}$ luminescence seems to disappear in doped crystals. It is worth noting that in undoped $\mathrm{YAlO}_{3}$, an yttrium analog of LuAP, bands corresponding to our 220 and $280 \mathrm{~nm}$ emissions have been observed and investigated quite intensively in the past [7-9].

In Fig. 3 we present RT excitation spectrum of $\mathrm{Ce}^{3+}$ luminescence in 0.11 mole\% Ce doped LuAP. Bands at 215 and $230 \mathrm{~nm}$ in addition to those mentioned previously (at 275,292 and $310 \mathrm{~nm}$ ) are typical of the $f$-to- $d$ transitions of the $\mathrm{Ce}^{3+}$ ion [1]. The intense excitation peak at $153 \mathrm{~nm}$ can be associated either with creation of band excitons or free electron-hole pairs, and consequently gives us an approximate evaluation of LuAP band gap as $8.1 \mathrm{eV}$. The broad band 


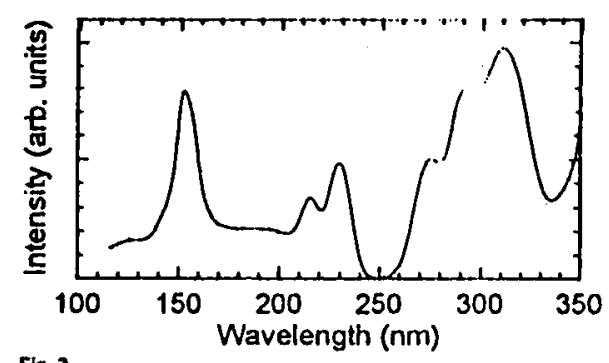

Fig. 3

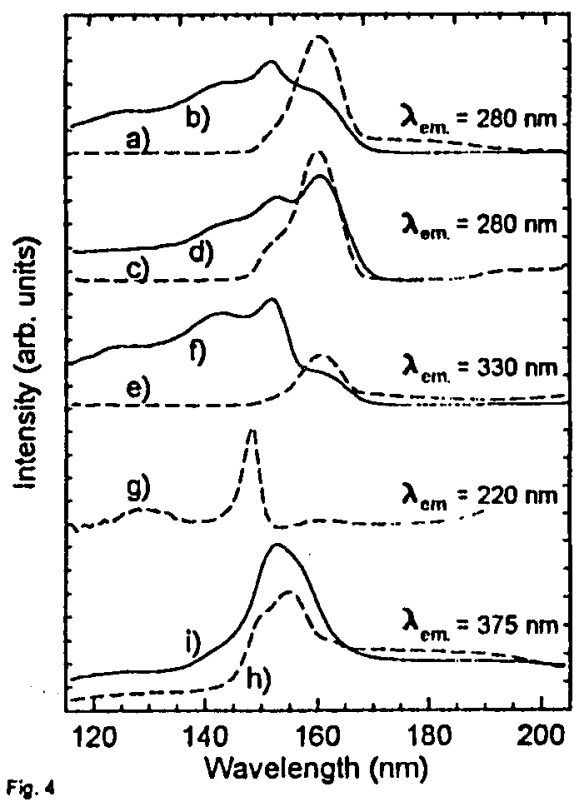

Fig. 3. Room temperature excitation spectrum of $\mathrm{Ce}^{3+}$ luminescence of $0.11 \mathrm{~mole} \%$ Ce doped LuAP.

Fig. 4. Excitation spectra of 0.11 mole\% Ce doped and undoped LuAP. Solid and dashed lines represent spectra measured at RT and $13 \mathrm{~K}$, respectively. Traces: $a, b, e$, $f$, and $g$ represent spectra of undoped sample, while $c, d, i$, and $h-$ Ce doped.

centered at about $180 \mathrm{~nm}$, partially overlapping both $153 \mathrm{~nm}$ and higher energy $\mathrm{Ce}^{3+} f-d$ bands, is most likely due to photoionization of $\mathrm{Ce}^{3+}$ ions.

Excitation spectra in the vicinity of band gap energies of Ce-doped and nominally undoped specimens are compared in Fig. 4. Traces $a$ and $b$ present excitation spectra of the $280 \mathrm{~nm}$ emission of undoped LuAP at $13 \mathrm{~K}$ and RT, respectively. Although the low-temperature (LT) spectrum consists of three bands, only 160 and $153 \mathrm{~nm}$ bands are in fact attributable to the $280 \mathrm{~mm}$ luminescence. The band centered at about $175 \mathrm{~nm}$ on the contrary belongs to $260 \mathrm{~nm}$ luminescence overlapping the $280 \mathrm{~nm}$ emission. The room temperature spectrum exhibits a strongly increased contribution of $153 \mathrm{~nm}$ band and new higher energy bands at about 142 and $125 \mathrm{~nm}$, which suggests presence of thermally activated processes. Low-temperature excitation spectrum of $280 \mathrm{~nm}$ emission in $0.11 \mathrm{~mole} \%$ Ce doped sample $(c)$ is nearly identical to that of undoped sample. The only difference is lack of the $175 \mathrm{~nm}$ band (and consequently the $260 \mathrm{~nm}$ emission). A corresponding RT spectrum $d$ consists of similar bands observed previously for undoped sample, but ratios of their intensities are different. In general the intensities of higher energy bands are decreased in comparison with the $160 \mathrm{~nm}$ band. Assuming that absorption of the $160 \mathrm{~nm}$ light creates band excitons, and the $153 \mathrm{~nm}$ light generates free $e-h$ pairs, it becomes evident that presence of Ce in the LuAP lattice does not 
affect direct creation of free excitons, but to some extent prevents their formation from free band charge carriers. The mechanism of this "prevention" is most probably a competition for holes and electrons by $\mathrm{Ce}^{3+}$ ions which strongly supports scintillation model based on consecutive carrier trapping proposed by Wojtowicz in [10].

Traces $e$ and $f$ present excitation spectra of $330 \mathrm{~nm}$ emission in undoped sample at $13 \mathrm{~K}$ and RT, respectively. Since the $330 \mathrm{~nm}$ luminescence overlaps the long-wavelength slope of $280 \mathrm{~nm}$ band, the measured spectra exhibit and mix features characteristic of both emissions. Some evaluations are however possible. The LT spectrum shows an excitation band centered at $217 \mathrm{~nm}$ which definitely belongs only to $330 \mathrm{~nm}$ emission ( $217 \mathrm{~nm}$ band is beyond the wavelength range of Fig. 4, but its existence is confirmed by Fig. $2 e$ ). The $160 \mathrm{~nm}$ light probably does not excite $330 \mathrm{~nm}$ emission (or at least does it insignificantly in comparison with excitation of $280 \mathrm{~nm}$ band), but shorter wavelengths again do it - the $148 \mathrm{~nm}$ excitation (Fig. $2 g$ ) gives the 280 and $330 \mathrm{~nm}$ bands at similar intensity level (very weak yet). The RT excitation spectrum of $330 \mathrm{~nm}$ emission consists exclusively of bands observed previously for $280 \mathrm{~nm}$ emission, but shorter wavelength bands are more intense in comparison with this at $160 \mathrm{~nm}$. In fact, if we subtract from this spectrum tliat measured for $280 \mathrm{~nm}$ emission, the result will not contain $160 \mathrm{~nm}$ band. This suggests that the origin of $330 \mathrm{~nm}$ luminescence may be entirely different from that of $280 \mathrm{~mm}$ one. While a large Stokes shift $(\approx 3.3 \mathrm{eV})$ affirm trapped excitons as a source of the latter, the former may be caused by defect or impurity centers which can be excited themselves (at $217 \mathrm{~nm}$ ) or act as radiative recombination centers.

The LT excitation spectrum of $220 \mathrm{~nm}$ emission of undoped LuAP $(g)$ consists of two weak bands at 160 and $128 \mathrm{~nm}$ and an intense narrow band peaking at $148 \mathrm{~nm}$. The $160 \mathrm{~nm}$ band is most probably artificial as a result of slight overlapping of emissions 220 and $280 \mathrm{~nm}$. The slim shape of the $148 \mathrm{~nm}$ peak, together with a large Stokes shift $(\approx 2.7 \mathrm{eV})$ proves the excitonic origin of the $220 \mathrm{~nm}$ luminescence. At RT the $220 \mathrm{~nm}$ band becomes unmeasurable. It is hard to believe that thermal quenching of the emission is responsible for that, more likely a low dissociation energy of excitons (before relaxation) is the reason. In Ce doped samples both $220 \mathrm{~nm}$ emission and its excitation spectra behave in the same way. In order to complete a comparison we show the short wavelength parts of $\mathrm{Ce}^{3+}$ emission excitation spectra at $13 \mathrm{~K}(h)$ and $\mathrm{RT}(i)$. Both spectra have a similar shape with major band at about $153 \mathrm{~nm}$, some contribution from photoionization, $160 \mathrm{~nm}$, and higher energy bands. Note that an admixture of the $160 \mathrm{~nm}$ band is greater at $13 \mathrm{~K}$, while the $153 \mathrm{~nm}$ band is much more intense at RT. An explanation of this beliavior can be presumably found in prolonged lifetime of excitons and reduced mobility of band holes at low temperature.

In Fig. 5 we present selected luminescence pulse shapes under a synchrotron light excitation. Previous experiments with $\gamma$ excitation resulted in scintillation pulse shape with an ummeasurable rise time (below $1 \mathrm{~ns}$ ) followed by prompt decay with main time constant about $18 \mathrm{~ns}$ for both Ce-doped and nominally undoped samples of LuAP. The contribution of longer components was below $5 \%$ of zero-time amplitude. No faster components have been found. The decay 
curve for Ce-doped sample has been published in [1]. An optical excitation in $\mathrm{Ce}^{3+} d-f$ absorption bands (a) gives a pulse shape with no rise time and a single exponential decay with time constant equal (within an experimental error) to that measured under $\gamma$ excitation. For 0.11 mole\% Ce doped sample this remains true even for excitation in band gap absorption region. Traces $b$ and $c$ represent pulse shapes of $280 \mathrm{~nm}$ luminescence in undoped specimen at $13 \mathrm{~K}$ and RT, respectively. Both are characterized by single exponential decays; there is, however, a slight possibility that very long components may be hidden in the background. The difference of decay times ( $4.0 \mathrm{~ns}$ at $13 \mathrm{~K}$ and $3.1 \mathrm{~ns}$ at RT) indicates some thermal quenching of $280 \mathrm{~mm}$ emission. Room temperature decay times of the same emission in $0.035(d)$ and 0.11 mole\% Ce doped (e) samples are 2.9 and $2.6 \mathrm{~ns}$, respectively, which indicate some quenching of the excitonic emission in presence of significant amounts of $\mathrm{Ce}$ in the LuAP lattice. Although this quenching most likely happens by nonradiative energy transfer to $\mathrm{Ce}^{3+}$ ions, it is too weak to be of importance for $\mathrm{Ce}^{3+}$ excitation.

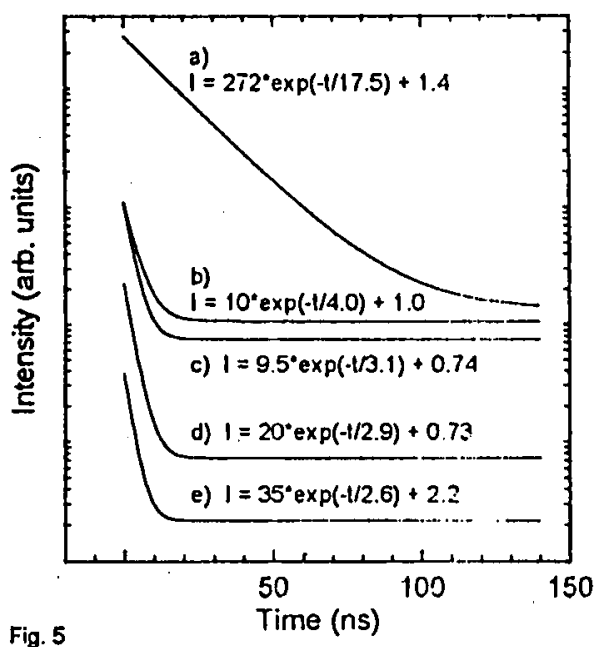

Fig. 5

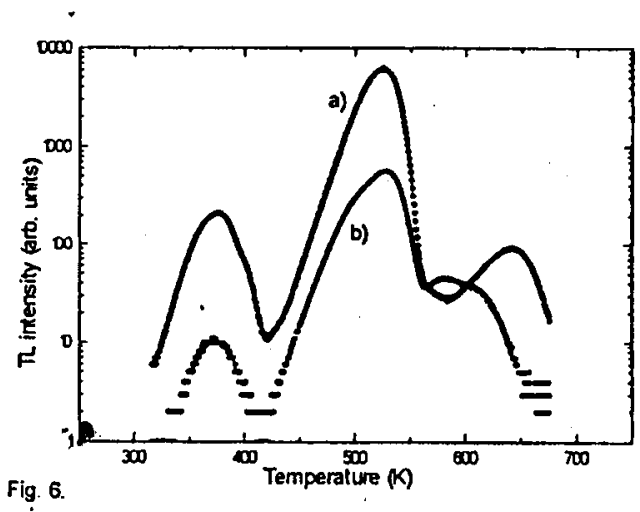

Fig. 5. Luminescence pulse shapes of $\mathrm{Ce}^{3+}(a)$ and $280 \mathrm{~nm}(b-e)$ emission under optical excitation: $a-292 \mathrm{~nm}$, RT, 0.11 mole\% Ce doped LuAP, $b-161 \mathrm{~nm}, 13 \mathrm{~K}$, undoped sample, $c-161 \mathrm{~nm}$, RT, undoped sample, $d-161 \mathrm{~nm}, \mathrm{RT}, 0.035 \mathrm{~mole} \%$ Ce doped LuAP, and $e-161 \mathrm{~nm}, \mathrm{RT}, 0.11 \mathrm{~mole} \%$ Ce doped LuAP.

Fig. 6 Glow-curves of LuAP following $\gamma$ irradiation: $a-0.11$ mole\% Ce doped, $b-$ undoped.

In Fig. 6 glow-curves of Ce-doped $a$ and undoped $b$ LuAP are presented. At a heating rate of $6 \mathrm{~K} / \mathrm{s}$, following $\gamma$-ray irradiation, both glow-curves have three peaks, two common at about 380 and $530 \mathrm{~K}$, and third at $640 \mathrm{~K}(\mathrm{a})$ or $580 \mathrm{~K}(b)$. The thermoluminescence (TL) signal is however much weaker for the undoped sample, which suggests a correlation between presence of $\mathrm{Ce}$ and the 
number of traps. The series of experiments employing an X-ray tube and a Hg lamp as irradiators was also performed. Regardless of the irradiation source similar glow peaks were observed. Yet, after the IIg lamp irradiation, the TL signal was much weaker. Using filters the $250 \mathrm{~nm}$ IIg line was recognized as responsible for sample excitation. While energy of absorbed photons is too low to stimulate band-to-band transitions, it is sufficient to release electrons from deeper traps. These electrons can then be retrapped into shallower traps. The shapes of TL emission spectra presented in Fig. 7 resemble those of X-ray excited luminescences, except that the relation of intensities is different. The TL signal of undoped sample (b) consists of
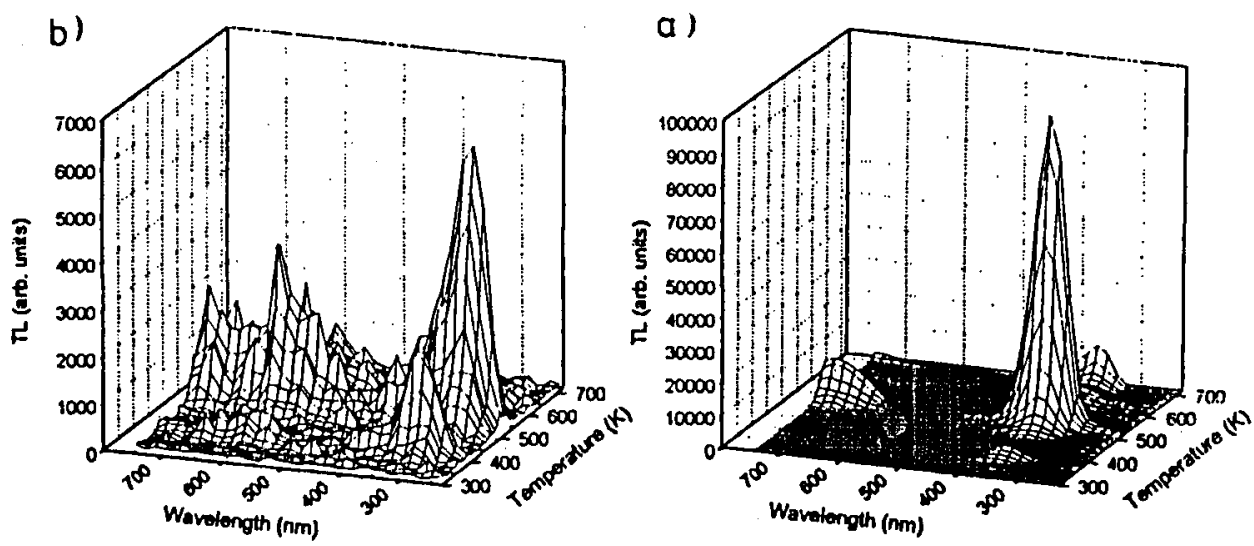

Fig. 7. TL emission spectra of LuAP following X-ray irradiation: (a) 0.11 mole\% Ce doped, (b) undoped.

a very weak host emission while the spectrum of doped one $(a)$ is dominated by a strong emission of $\mathrm{Ce}^{3+}$ ions. Assuming that the simple classic model of TL [11] is true for LuAP, one can assert that $\mathrm{Ce}^{3+}$ ions effectively trap holes during the irradiation stage and then, gaining a positive potential, easily attract conduction band electrons. A more detailed description of TL processes in LuAP, including estimations of trap depths and frequency factors, will be published elsewhere.

\section{Conclusions}

Although there was no entirely Ce-free sample available, the LuAP lattice emission has been identified. The excitonic $280 \mathrm{~nm}$ band was recognized as a main component of this emission. The comparison of decay times of $280 \mathrm{~nm}$ luminescence at various Ce doping levels demonstrated only slight quenching of host emission, definitely not suflicient to account for nomradiative energy transfer as a significant source of cerium excitation. Despite of obvious signs of reabsorption of host emission by $\mathrm{Ce}^{3+}$ ions, no vital consequences of that were found in scintillation pulse shapes. Excitation spectra indicate competition for free charge carriers between $\mathrm{Ce}$ and host emissions, $\mathrm{Ce}^{3+}$ being a winner of that contest.

Theoretical speculations presented in [10] lead to the conclusion that $\mathrm{Ce}^{3+}$ ions in wide band gap materials will act as hole traps much more likely than as 
electron traps. It was confirmed by thermoluminescence spectra which showed that $\mathrm{Ce}^{3+}$ ion is a very efficient hole trap and works perfectly as radiative recombination center. All the above observations, together with an astonishing Ce capability to collect excitation deposited in the crystal lattice by $\gamma$ photons in the form of hot band charge carriers, let us conclude that scintillation of Ce : LuAP is accomplished by consecutive trapping first band holes and then electrons by $\mathrm{Ce}^{3+}$ ions.

\section{Acknowledgments}

We acknowledge the support of the European Economic Community (Contract No. ERBCIPDCT940037), the US Dept. of Energy (grant no. DE-FG-0290ER61033) and the Netherlands Technology Foundation (STW). We thank Dr. J.C. Sutherland and his group for their hospitality and assistance in performing experiments at the BNSLS.

\section{References}

[1] A. Lempicki, M.H. Randles, D. Wiśniewski, M. Balcerzyk, C. Brecher, A.J. Wojtowicz, IEEE Nucl. Sci. 42, 280 (1995).

[2] M. Moszynski, D. Wolski, T. Ludziejewski, A. Lempicki, C. Brecher, D. Wiśniewski, A.J. Wojtowicz, in: Proc. Int. Conf. on Inorganic Scintillators and their Applications, SCINT95, Delft 1995, Eds. P. Dorenbos, C.W.E. van Eijk, Delft University Press, Delft (The Netherlands) 1996, p. 348.

[3] A. Lempicki, C. Brecher, D. Wiśniewski, E. Zycl, in: Proc. Int. Conf. on Inorganic Scintillators and their Applications, SCINT95, Delft 1995, Eds. P. Dorenbos, C.W.E. van Eijk, Delf University Press, Delf (The Netherlands) 1996, p. 340.

[4] A. Lempicki, C. Brecher, D. Wiśniewski, E. Zych, A.J. Wojtowicz, Nucl. Sci. Symp. 6Med. Imag. Conf., San Francisco 1995, to be published in Conference Record and in IEEE Trans. Nucl. Sci.

[5] T.M. Piters, Ph.D. Thesis, Delft University of Technology, Delft 1993, p. 49.

[6] B. van den Burg, Report no. 95, Delft University of Technology, Delft 1995, p. 11.

[7] R.L. Wood, W. Hayes, J. Phys. C, Solid State Phys. 15, 7209 (1982).

[8] A.I. Kuznetsov, V.N. Abramov, B.R. Namozov, T.V. Uibo, Trudy Instituta Fiziki AN Estonskioj SSR 53, 83 (1995).

[9] A.I. Kuznetsov, B.R. Namozov, V.V. Myurk, Sov. Phys. Solid State 27, 1819 (1985).

[10] A.J. Wojtowicz, in: Proc. Int. Conf. on Inorganic Scintillators and their Applications, SCINT95, Delft 1995, Eds. P. Dorenbos, C.W.E. van Eijk, Delft University Press, Delft (The Netherlands) 1996, p. 95.

[11] S.W.S. McKeever, Thermoluminescence of Solids, Cambridge University Press, Cambridge 1985. 